\title{
Strafrecht und Medizin
}

\section{Kurt Seelmann}

1 Donatsch A, Blocher F, Hubschmid Volz A (Hrsg.). Strafrecht und Medizin. Tagungsband des Instruktionskurses der Schweizerischen Kriminalistischen Gesellschaft vom 26./27. Oktober 2006 in Flims. Bern: Stämpfli; 2007. 137 Seiten.

\section{Korrespondenz:}

Prof. Dr. iur. Kurt Seelmann

Universität Basel

Juristische Fakultät

Peter Merian-Weg 8

CH-4002 Basel

kurt.seelmann@unibas.ch
Einige Referate im Rahmen eines Gedankenaustausches zwischen Strafrechtlern und Medizinern waren die Grundlage für diesen Band über aktuelle Probleme im Verhältnis der beiden Disziplinen [1]. Brigitte Tag, Strafrechtsprofessorin an der Universität Zürich, geht auf das schwierige Verhältnis von Ethik und Recht im Rahmen der Medizin ein. Sie macht deutlich, dass es im Medizinrecht für Juristen unvermeidlich ist, auch auf Fragen der Ethik einzugehen. Ärztliche Entscheidungen ebenso wie Patientenentscheidungen würden angesichts neuer Möglichkeiten, aber auch neuer Kostenprobleme schwieriger, zugleich änderten sich aber die grundlegenden - bisher eher paternalistischen - Regeln ärztlicher Tätigkeit. Rechtsnormen stehen oft in einem schwer durchschaubaren Spannungsverhältnis aus kantonalen Spezialgesetzen, OR, ZGB und StGB, aber auch - teilweise in Gesetzesrang erhobenen - untergesetzlichem «soft law» wie Richtlinien der FMH, der SAMW und der Good Clinical Practice.

Der Berner Rechtsmediziner Ulrich Zollinger thematisiert die Schwierigkeiten bei der interdisziplinären Zusammenarbeit von Medizinern und Juristen. Ärzten seien ihre Rechte und Pflichten oft nicht hinreichend bekannt, und zudem gingen beide Professionen sehr unterschiedlich mit Kritik um: Für Juristen sei Kritik allein schon im gerichtlichen Instanzenzug ganz gewöhnlich, Medizinern sei Kritik eher ungewohnt. Ärzte überschätzen oft die Reichweite der Schweigepflicht oder glaubten, Patienten vor der Justiz schützen zu müssen, Juristen gäben den Ärzten oft nicht hinreichend Informationen und achteten in der Formulierung ihrer Fragen an Ärzte zuwenig auf die Grenzen der Disziplinen.

Der Zürcher Leitende Oberstaatsanwalt Andreas Brunner befasst sich speziell mit dem Arzt in den Mühlen der Justiz am Beispiel des Behandlungsfehlers, den er von der Behandlungskomplikation abzugrenzen sucht. Er bemängelt zu Recht, dass es an einer Statistik von Behandlungsfehlern mangele und nur nichtrepräsentative Daten von FMH-Gutachterstellen vorlägen. Eine gesamtschweizerische Lösung sei etwa bei der Meldepflicht für ungewöhnliche Todesfälle anzustreben, auch seien eine Professionalisierung der Leichenschau und eine Spezialisierung in den Staatsanwaltschaften wünschenswert. Als

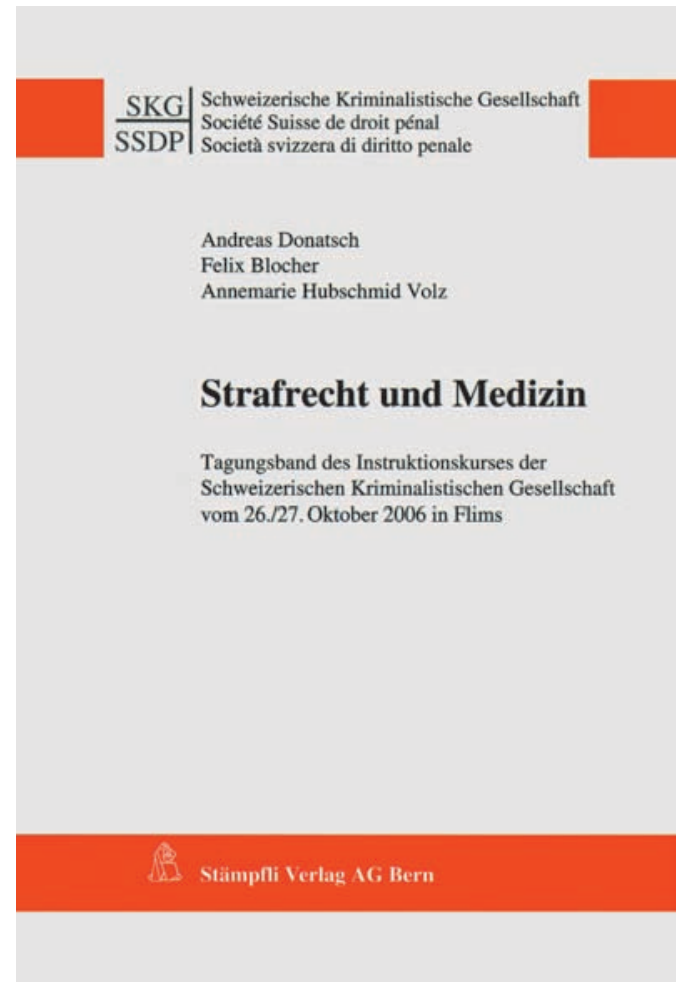

vorteilhaft erscheint ihm auch ein System von «Critical Incident Reporting» (CIRS), also ein System der Meldung von Beinaheschäden.

Bundesrichter Hans Wiprächtiger stellt die Rechtsprechung des Bundesgerichts zum Arztfehler vor. In den letzten 20 Jahren sei eine erhebliche Zunahme strafrechtlich relevanter Vorwürfe gegen die Ärzteschaft zu verzeichnen, verursacht durch eine starke Juridifizierung des Arzt-Patienten-Verhältnisses. Allerdings sei zu beachten, dass der Grossteil solcher Vorwürfe bereits auf der Ebene der Untersuchungsbehörden eingestellt werde. Wiprächtiger plädiert für die Beibehaltung der Rechtsprechung, wonach auch die Heilbehandlung eine tatbestandsmässige Körperverletzung sein könne, da sonst Strafbarkeitslücken entstünden. Am Beispiel der ex ante vorzunehmenden Bestimmung der Sorgfaltspflicht macht er aber auch deutlich, dass die Ärztin oder der Arzt mit vertretbaren Handlungen oder Unterlassungen kein Strafbarkeitsrisiko eingingen.

Der Basler Anästhesie-Chefarzt Daniel Scheidegger stellt ein Modell der Fehlermeldung in anonymer Form vor, das u. a. an der Universität 
Basel für die Anwendung in der gesamten Schweiz entwickelt worden ist. Er stellt klar, dass mit dem Begriff des «Fehlers» zunächst keineswegs ein Schuldvorwurf verbunden ist, da auch schon die objektive Ungeeignetheit einer Vorgehensweise ein Fehler sein könne. Zu Recht sieht er aber ein zentrales juristisches Problem dann, wenn ein vorwerfbarer Fehler zur Schädigung bei einem Menschen führt. Die Vorteile des aus der Luftfahrt bekannten anonymen Fehlermeldesystems, das einen starken Anreiz zur Meldung von Fehlern und damit zum Lernen aus Fehlern bietet, kollidiert dann mit dem gleichfalls berechtigten Interesse an der rechtlichen Erfassung und Aufarbeitung von Schädigungen.

Der auch bei medizinischen Eingriffen relevanten Unterscheidung von Drittschädigung mit Einwilligung und Selbstgefährdung widmet sich der Zürcher Strafrechtsprofessor Andreas Donatsch. Selbstgefährdung ist nicht strafbar und deshalb auch nicht die Gehilfenschaft dazu; eine Tötung durch Dritte ist demgegenüber nach geltendem Recht auch mit Einwilligung strafbar das macht die praktische Bedeutung der Unterscheidung aus. Donatsch differenziert mit Lehre und Rechtsprechung dahingehend, dass Selbstgefährdung eine Beherrschung des Tatgeschehens durch den sich selbst Gefährdenden vor- aussetze. Er räumt aber auch ein, dass in der Praxis Kombinationen aus beiden rechtlichen Figuren denkbar seien, etwa wenn der Patient nach gebührender Aufklärung in zwei mögliche Operationsmethoden sich für die riskantere, aber bei Gelingen mehr erfolgversprechende entscheidet.

Der Berner Rechtsmediziner Michael Thali macht zum Abschluss mit «Virtopsy» bekannt, einer 3-D-Dokumentationsmethode für Körperoberflächen und (auf den Körper einwirkenden) Objekten, aber auch für eine nichtinvasive 3-DDarstellung des Körperinneren. Letzteres mache möglicherweise in Zukunft eine Leichenöffnung auch zu gerichtsmedizinischen Zwecken gänzlich überflüssig. Ein Bildanhang gibt einen anschaulichen Überblick über die damit vorhandenen Möglichkeiten.

Insgesamt erlaubt der überschaubare und auf wesentliche Fragen konzentrierte Band einen raschen Überblick über die aktuellen Probleme im Verhältnis von Strafrecht und Medizin. Sein Vorteil ist auch, dass die vorhandenen Spannungen in diesem Verhältnis von beiden Seiten her mit Verständnis und wechselseitigem Einfühlungsvermögen beleuchtet werden. Die Lektüre kann deshalb Juristen wie Medizinern empfohlen werden. 